\title{
A NORMALIZATION-PROCEDURE FOR THE FIRST ORDER CLASSICAL NATURAL DEDUCTION WITH FULL LOGICAL SYMBOLS
}

\author{
By \\ ANDOU, Yuuki
}

\section{§ 0. Introduction}

The system of natural deduction was introduced by Gentzen [1]. He also introduced the system of sequent calculus in order to prove his Hauptsatz which states every proof can be reduced to a proof without roundabouts. (In some cases, the Hauptsatz is called the cut-elimination theorem or the normalization theorem.) His system of natural deduction was not suitable for the Hauptsatz in the case of classical logic, because in the system the classical logic was formalized as the intuitionistic logic with the law of the excluded middle. Prawitz [2], [3] settled this trouble in his system of natural deduction by formalizing the classical logic as the minimal logic with classical absurdity rule. However his solution was a partial one, since his system of classical logic did not have the logical symbols for the disjunction and for the existential quantifier as the primitive logical symbols. Seldin [4], [5] and Stålmarck [6] proved the normalization theorem for the first order classical natural deduction with full logical symbols. But the reduction procedures defined by them are complicated in comparison with Prawitz's one.

In this paper, we define another reduction procedure for the first order classical natural deduction with full logical symbols. It is as simple as Prawitz's one is. In other words, our reduction procedure is a natural extension of the Prawitz's. Our proof of the normalization theorem will be done simultaneously for the intuitionistic logic and for the classical logic, as the Gentzen's proof of the Hauptsatz was. Notice that our normalization theorem is one of the so called weak normalization theorems.

\section{§ 1. System}

The system used in this paper is the first order classical logic formalized in the style of natural deduction. It has all logical symbols as primitive ones. 
The inference rules consist of the introduction rule and elimination rule for each logical symbol, and the classical absurdity rule [2]. These are denoted by $(\mathcal{L} I)$ and $(\mathcal{L} E)$ for each logical symbol $\mathcal{L}$, and $(\perp c)$ respectively. We present them by the inference figure schemata in the same manner with Gentzen [1].

$$
\begin{aligned}
& \text { (\&I) } \frac{\mathfrak{A}_{1} \mathfrak{A}_{2}}{\mathfrak{A}_{1} \& \mathfrak{A}_{2}} \quad \text { (\&E) } \frac{\mathfrak{A}_{1} \& \mathfrak{A}_{2}}{\mathfrak{A}_{i}} \quad(i=1 \text { or } 2) \\
& (\vee I) \frac{\mathfrak{A}_{i}}{\mathfrak{A}_{1} \vee \mathfrak{A}_{2}}(i=1 \text { or } 2) \quad(\vee E) \frac{\mathfrak{A}_{1} \vee \mathfrak{A}_{2}}{\mathfrak{S}}\left[\begin{array}{c}
{\left[\mathfrak{U}_{1}\right]} \\
\mathbb{S}
\end{array}\right. \\
& \text { [थ] }
\end{aligned}
$$

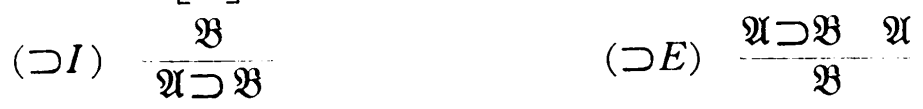

$$
\begin{aligned}
& \text { [ } \mathfrak{X}] \\
& \text { (フI) } \frac{1}{7 \mathfrak{U}} \\
& (>E) \frac{>\mathfrak{A} \mathfrak{A}}{\perp} \\
& (\forall I) \frac{\mathfrak{q} \mathfrak{a}}{\forall \mathfrak{x} \mathfrak{q} \mathfrak{r}} \\
& (\forall E) \frac{\forall \mathfrak{x} \mathfrak{q} \mathfrak{x}}{\mathfrak{F} \mathfrak{t}} \\
& (\exists I) \quad-\frac{\mathfrak{F}^{t}}{\exists \mathfrak{x} \mathfrak{F} x} \\
& (\exists E) \frac{\exists \mathfrak{x} \mathfrak{F} \mathfrak{r} \quad(5}{\mathfrak{S}}
\end{aligned}
$$

$(\forall I)$ and $(\exists E)$ are subject to the restriction of eigenvariable [1]. In a proof, the eigenvariables must be separated as usual [2].

\section{§2. Definitions}

DEFINITION (Maximum formula). Let $\mathfrak{A}$ be a formula-occurrence in a proof $\Pi$. $\mathscr{U}$ is a maximum formula in $\Pi$ iff it satisfies the following conditions.

(1) $\mathfrak{A}$ is not an assumption-formula. And the inference rule whose conclusion is $\mathfrak{A}$ is an introduction rule, a $(\vee E)$, a $(\exists E)$, or a $(\perp c)$.

(2) $\mathfrak{U}$ is the major premiss of an elimination rule.

Definition (Normal proof). A proof $\Pi$ is normal iff it contains no maximum formula.

DEFINITION (Regular proof). In a proof-figure, an assumption-formula discharged by a $(\perp c)$ is regular iff it is the major premiss of a $(7 E)$. A prooffigure is regular iff any assumption-formula discharged by any $(\perp c)$ in the proof 
is regular.

DEFINITION (Segment). Let $\mathfrak{A}_{1}, \cdots, \mathfrak{A}_{n}$ be a sequence of formula-occurrences in a regular proof $\Pi . \mathfrak{A}_{1}, \cdots, \mathfrak{A}_{n}$ is a segment in $\Pi$ iff it satisfies the following conditions.

(1) $\mathfrak{A}_{1}$ is neither the conclusion of a $(\vee E)$ nor that of a $(\exists E)$. Moreover, $\mathfrak{A}_{1}$ is not the conclusion of a $(\perp c)$ where at least one assumption formula is discharged.

(2) For all $i<n$; (a) $\mathfrak{A}_{i}$ is a minor premiss of a $(\vee E)$ or a $(\exists E)$, and the conclusion of the inference is $\mathfrak{A}_{i+1}$; or (b) $\mathfrak{A}_{i}$ is the minor premiss of a $(\neg E)$ whose major premiss is an assumption-formula discharged by a $(\perp c)$, and the conclusion of the $(\perp c)$ is $\mathfrak{A}_{i+1}$.

(3) $\mathfrak{A}_{n}$ is neither a minor premiss of a $(\vee E)$ nor that of a $(\exists E)$. Moreover, $\mathfrak{U}_{n}$ is not the minor premiss of a $(>E)$ whose major premiss is an assumption-formula discharged by a $(\perp c)$.

\section{§3. Reduction steps}

To simplify the description, our reduction steps are defined only for regular proofs. For non regular proofs, we use the following lemma.

LEMMA 1. Let II be a given non regular proof. Then we can construct a regular proof $\Pi^{\prime}$ which has the same set of assumptions and the same end formula with $\Pi$.

ProOF. Let $7 \mathfrak{A}$ be a non regular assumption-formula in $\Pi$. Then, transform $\Pi$ by replacing $>\mathfrak{A}$ with the following subproof:

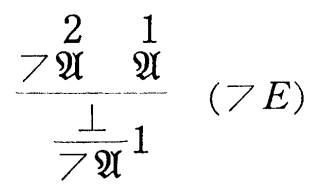

Where, $\frac{1}{\mathfrak{Q}}$ is discharged by the $(>I)$ represented in the above figure with the indicator 1 . And $>\underset{\mathfrak{A}}{2}$ is discharged by the $(\perp c)$ which corresponds with the $(\perp c)$ in $\Pi$ discharging the $>\mathfrak{A}$ in $\Pi$. Then $\underset{2}{2}$ is regular. Clearly this transformation does not change the set of assumptions and the end formula. By applying this transformation for all non regular assumption-formulae of all $(\perp c) \mathbf{s}$ in $\Pi$, we get the regular proof: $\Pi^{\prime}$.

Now, we define our reduction steps. Let $\Pi$ be a regular but not normal 
proof. And let $\mathfrak{M}$ be a maximum formula in $\Pi$, and $I$ be the inference whose conclusion is $\mathfrak{M}$. The reduction of $I$ at $\mathfrak{M}$ is defined according to $I$.

First we treat the case that $I$ is a $(\perp c)$. Let $K_{1}, \cdots, K_{n}$ be all the $(>E)$ s whose major premisses are discharged by $I$, if they exist. We show $\Pi$ by the next figure.

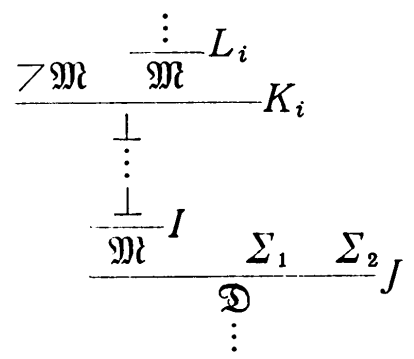

where $\Sigma_{1}$ and $\Sigma_{2}$ are the proofs of the minor premisses of $J$, if they exist. The reduction is carried out as follows:

(1) For all $i$, replace the inference $K_{i}$ by the following figure.

$$
>\mathfrak{D} \frac{\overline{\mathfrak{M}} L_{i} \Sigma_{1} \Sigma_{2}}{\perp} J
$$

(2) Concatenate the premiss of $I$ with the conclusion of $J$ by a $(\perp c)$ where the $7 \mathfrak{D}$ 's brought about by the replacement (1) are discharged.

Notice that there is no assumption formula discharged by $I$, except for the major premisses of $K_{1}, \cdots, K_{n}$; because $\Pi$ is regular. The next diagram shows the reduction mentioned above.

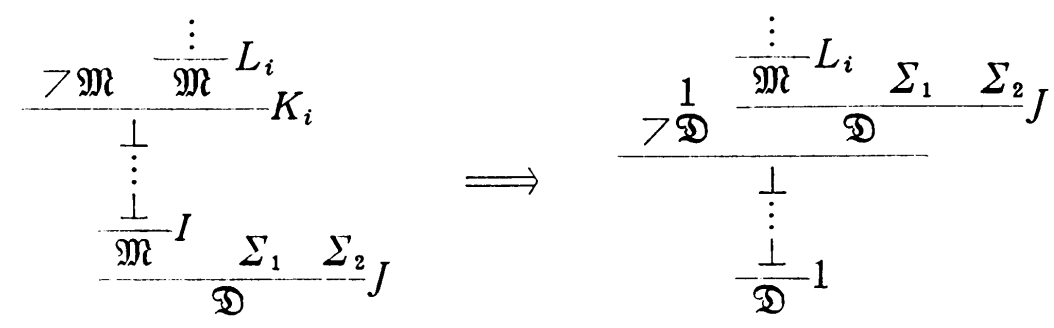

In the other cases, i.e. $I$ is an introduction rule, a $(\vee E)$, or a $(\exists E)$; the reduction steps are the same with the ones for the intuitionistic logic, defined by Prawitz [2], [3]. We show them briefly by the figures below.

(i) $I$ is a $(\& I)$ :

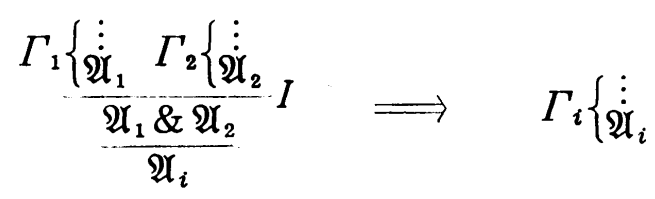


where $\mathfrak{A}_{1} \& \mathfrak{U}_{2}$ is the maximum formula: $\mathfrak{M}$.

(ii) $I$ is a $(\forall I)$ : similarly to the case (i).

(iii) $I$ is a $(\vee I)$ :

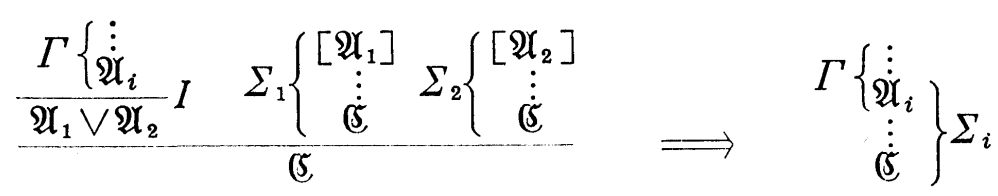

where $\mathfrak{A}_{1} \vee \mathfrak{A}_{2}$ is the maximum formula: $\mathfrak{M}$.

(iv) $I$ is a $(\exists I)$ : similarly to the case (iii).

(v) $I$ is a $(\supset I)$ :

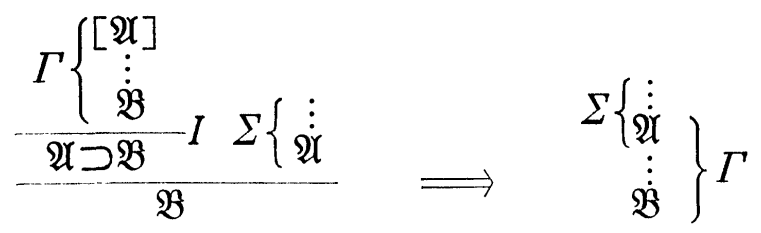

where $\mathfrak{A} \supset \mathfrak{B}$ is the maximum formula: $\mathfrak{M}$.

(vi) $I$ is a $(>I)$ : similarly to the case (v).

(vii) $I$ is a $(\vee E)$ :

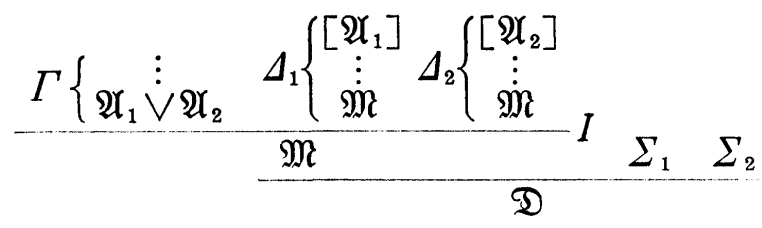

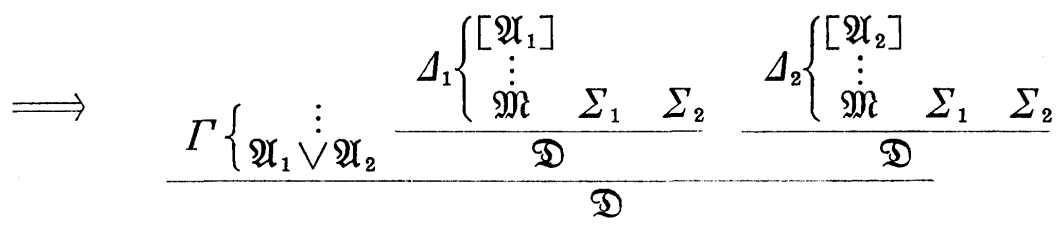

(viii) $I$ is a $(\exists E)$ : similarly to the case (vii).

Our reduction steps are all defined by the items mentioned above. It is clear that the following fact holds.

FACT 2. The proof which is obtained from a regular proof by applying our reduction step is also regular.

\section{§4. Proof of the normalization theorem}

Notations. Let $\mathfrak{A}$ be a maximum formula in a regular proof. By $g(\mathfrak{A})$ we denote the number of the logical symbols occurring in $\mathfrak{A}$. By $r(\mathfrak{A})$ we denote the maximum length of the segments whose last formula is $\mathfrak{A}$. By $l(\mathfrak{A})$ we denote the number of inferences below $\mathfrak{A}$ in the proof. 
DEFINITION (Degree of a maximum formula). Let $\mathfrak{A}$ be a maximum formula in a regular proof. The degree of $\mathfrak{A}$, denoted by $d(\mathfrak{A})$, is the ordered pair defined as follows:

$$
d(\mathfrak{A})=\langle g(\mathfrak{A}), r(\mathfrak{U})\rangle
$$

Degrees of maximum formulae are compared by lexicographical order.

Notations. Let $\Pi$ be a regular proof. Notations $M(\Pi)$ and $E(\Pi)$ are defined as follows:

$M(\Pi)=\left\{\begin{array}{l}\langle 0,0\rangle, \quad \text { if } \Pi \text { is normal, } \\ \max \{d(\mathfrak{A}) \mid \mathfrak{A} \text { is a maximum formula in } \Pi\}, \quad \text { otherwise }\end{array}\right.$

$E(\Pi)=\{\mathfrak{A}:$ a maximum formula in $\Pi \mid d(\mathfrak{A})=M(\Pi)\}$

Definition (Degree of a proof). Let $\Pi$ be a regular proof. The degree of $\Pi$, denoted by $d(\Pi)$, is the ordered triple defined as follows:

$$
d(\Pi)=\left\langle M(\Pi), \text { Card } E(\Pi), \sum_{\mathfrak{M} \in E(\Pi)} l(\mathfrak{A})\right\rangle
$$

where in the case of $E(\Pi)$ is empty, by $\sum_{\mathfrak{A} \in E_{(}(\Pi)} l(\mathfrak{H})$ we mean 0 . Degrees of proofs are compared by lexicographical order.

We call a formula-occurrence $\mathfrak{A}$ a side-set formula of a formula-occurrence $\mathfrak{B}$, if $\mathfrak{A}$ is one of the minor premisses of the inference whose major premiss is $\mathfrak{B}$.

LEMMA 3. Let II be a given regular proof. If $\Pi$ is not normal, we can find in it a formula-occurrence $\mathfrak{A}$ which satisfies the following conditions.

(1) $\mathfrak{A} \in E(\Pi)$.

(2) If $\mathfrak{B} \in E(\Pi)$; and if $\mathcal{S}$ is a segment in $\Pi$, whose last formula is $\mathfrak{A}$; then $\mathfrak{B}$ is not above the first formula of $\mathcal{S}$.

(3) If $\mathfrak{B} \in E(\Pi)$; and if $\mathcal{S}$ is a segment in $\Pi$, whose last formula is $\mathfrak{B}$; then the first formula of $\mathcal{S}$ is not above nor equal to any of the side-set formulae of $\mathfrak{A}$.

PROOF. Construct a sequence $\mathfrak{A}_{1}, \mathfrak{A}_{2}, \cdots$ of maximum formulae in $\Pi$ by the following manner. Take $\mathfrak{A}_{1}$ from the maximum formulae satisfying the condition (1) and (2). If $\mathfrak{A}_{1}$ also satisfies the condition (3), terminate the sequence at it. If not, take $\mathfrak{A}_{2}$ from the maximum formulae destroying the condition (3) for $\mathfrak{A}_{1}$ and satisfying the condition (1) and (2). By iterating this construction, we obtain the sequence $\mathfrak{A}_{1}, \mathfrak{A}_{2}, \ldots$. It holds that if $m<n$ then $\mathfrak{A}_{m} \neq \mathfrak{A}_{n}$, by 
induction on $n-m$. Therefore, the sequence $\mathfrak{A}_{1}, \mathfrak{A}_{2}, \cdots$ is finite. Then, the last formula of the sequence satisfies all the conditions for $\mathfrak{A}$.

It is clear that the following fact holds.

FACT 4. Let $\mathfrak{A}$ be a formula-occurrence in a regular proof $\Pi$. If $\mathfrak{A}$ satisfies the conditions of Lemma 3, then it also satisfies the following condition.

(3') If $\mathfrak{B} \in E(\Pi)$, then $\mathfrak{B}$ is not above nor equal to any of the side-set formulae of $\mathfrak{A}$.

THEOREM (Normalization theorem). Let $\Pi$ be a given proof. Then we can construct a normal proof which has the same set of assumptions and the same end formula with $\Pi$.

Proof. By Lemma 1 and Fact 2, it can be assumed that $\Pi$ is regular. We prove this theorem by induction on the degree of $\Pi$. If $\Pi$ is not normal, we can find in $I I$ a formula-occurrence, say $\mathfrak{M}$, which is one of the maximum formulae satisfying the conditions for $\mathfrak{A}$ of Lemma 3 . Reduce $\Pi$ at $\mathfrak{M}$. Then, the degree of the proof obtained, say $\Pi^{\prime}$, is lower than that of $\Pi$. In the following we show this fact according to the inference, say $I$, whose conclusion is $\mathfrak{M}$.

Case $1 . \quad I$ is a $(\& I)$ or a $(\forall I)$ : Because $\mathfrak{M}$ satisfies the condition (1) for $\mathfrak{A}$ of Lemma 3, it holds that

$$
\langle M(\Pi), \text { Card } E(\Pi)\rangle\rangle\left\langle M\left(\Pi^{\prime}\right), \text { Card } E\left(\Pi^{\prime}\right)\right\rangle
$$

This leads $d(\Pi)>d\left(\Pi^{\prime}\right)$.

Case 2. $I$ is a $(\vee I)$ or a $(\exists I)$ : Because $\mathfrak{M}$ satisfies the conditions (1) and (2) for $\mathfrak{A}$ of Lemma 3 , it holds that

$$
\langle M(\Pi), \text { Card } E(\Pi)\rangle\rangle\left\langle M\left(\Pi^{\prime}\right), \text { Card } E\left(\Pi^{\prime}\right)\right\rangle
$$

This leads $d(\Pi)>d\left(\Pi^{\prime}\right)$.

Case 3. $I$ is a $(\supset I)$ or a $(>I)$ : Because $\mathfrak{M}$ satisfies the conditions (1) and $\left(3^{\prime}\right)$ for $\mathfrak{A}$ of Lemma 3 and Fact 4 , it holds that

$$
\langle M(\Pi), \text { Card } E(\Pi)\rangle\rangle\left\langle M\left(\Pi^{\prime}\right), \text { Card } E\left(\Pi^{\prime}\right)\right\rangle
$$

This leads $d(\Pi)>d\left(\Pi^{\prime}\right)$.

Case 4. $I$ is a $(\vee E)$, a $(\exists E)$, or a $(\perp c)$ : Let $J$ be the inference in $\Pi$ whose major premiss is $\mathfrak{M}$. Let $\mathfrak{D}^{1}$ be the formula-occurrence in $\Pi$ which is the conclusion of $J$. Let $\mathfrak{D}^{0}$ be the last formula of a segment in $\Pi$ which includes $\mathfrak{D}^{1}$ as its member. We show $\Pi$ by the next figure. 


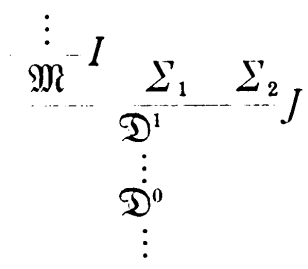

Case 4-1. $\mathscr{D}^{0}$ is not a maximum formula in $\Pi$ : Because $\mathfrak{M}$ satisfies the conditions (1) and $\left(3^{\prime}\right)$ for $\mathfrak{A}$ of Lemma 3 and Fact 4 , it holds that

$$
\langle M(\Pi), \text { Card } E(\Pi)\rangle\rangle\left\langle M\left(\Pi^{\prime}\right), \text { Card } E\left(\Pi^{\prime}\right)\right\rangle
$$

This leads $d(\Pi)>d\left(\Pi^{\prime}\right)$.

Case 4-2. $\mathfrak{D}^{0}$ is a maximum formula in $\Pi$ : It holds that $d\left(\mathfrak{D}^{0}\right)<M(\Pi)$, since ;

(a) If $J$ is a $(\vee E)$ or a $(\exists E)$, then there exists a segment in $\Pi$ whose first formula is above or equal to one of the side-set formulae of $\mathfrak{M l}$ and whose last formula is $\mathfrak{D}^{0}$. This leads $d\left(\mathfrak{D}^{0}\right)<M(\Pi)$, because $\mathfrak{M}$ satisfies the condition (3) for $\mathfrak{A}$ of Lemma 3.

(b) Otherwise, it holds that $g\left(\mathfrak{D}^{1}\right)<g(\mathfrak{M})$. This leads $d\left(\mathfrak{D}^{0}\right)<d(\mathfrak{M})=M(\Pi)$. Let $\tilde{\mathfrak{D}}^{0}$ be the maximum formula in $\Pi^{\prime}$ which corresponds with $\mathfrak{D}^{0}$. Then it holds that $d\left(\tilde{\mathfrak{D}}^{0}\right) \leqq M(\Pi)$, since $g\left(\tilde{\mathfrak{D}}^{0}\right)=g\left(\mathfrak{D}^{0}\right)$ and $r\left(\widetilde{\mathfrak{D}}^{0}\right) \leqq r\left(\mathfrak{D}^{0}\right)+1$.

Case 4-2-1. $\quad d\left(\widetilde{\mathfrak{D}}^{0}\right)<M(\Pi)$ : Because $\mathfrak{M}$ satisfies the conditions $(1)$ and $\left(3^{\prime}\right)$ for $\mathfrak{A}$ of Lemma 3 and Fact 4 , it holds that

$$
\langle M(\Pi), \text { Card } E(\Pi)\rangle\rangle\left\langle M(\Pi), \text { Card } E\left(\Pi^{\prime}\right)\right\rangle
$$

This leads $d(\Pi)>d\left(\Pi^{\prime}\right)$.

Case $4-2-2 . \quad d\left(\widetilde{\mathfrak{D}}^{0}\right)=M(\Pi)$ : For each $\mathfrak{B}$ in $E(\Pi)$, we define a maximum formula $\mathfrak{B}^{\prime}$ in $\Pi^{\prime}$ as follows :

(a) If $\mathfrak{P}$ is $\mathfrak{M}$, then $\mathfrak{P}^{\prime}$ is $\tilde{\mathfrak{D}}^{0}$.

(b) Otherwise, $\mathfrak{B}^{\prime}$ is the maximum formula in $\Pi^{\prime}$ which corresponds with $\mathfrak{P}$. (Since $\mathfrak{M}$ satisfies the condition $\left(3^{\prime}\right)$ for $\mathfrak{A}$ of Fact 4 , exactly one formula-occurrence in $\Pi^{\prime}$ corresponds with $\mathfrak{B}$.) For $d\left(\widetilde{\mathfrak{D}}^{0}\right)=M(\Pi)$, it holds that $E\left(\Pi^{\prime}\right)=\left\{\mathfrak{P}^{\prime} \mid \mathfrak{B} \in E(\Pi)\right\}$. Therefore,

$$
\langle M(\Pi), \text { Card } E(\Pi)\rangle=\left\langle M\left(\Pi^{\prime}\right), \text { Card } E\left(\Pi^{\prime}\right)\right\rangle
$$

Next, we compare $l\left(\mathfrak{P}^{\prime}\right)$ with $l(\mathfrak{P})$. If $\mathfrak{P}$ is $\mathfrak{M}$, then $l(\mathfrak{P})>l\left(\mathfrak{P}^{\prime}\right)$; since $\mathfrak{M}$ is is above $\mathfrak{D}^{0}$. Otherwise, $l(\mathfrak{B}) \geqq l\left(\mathfrak{P}^{\prime}\right)$; since $\mathfrak{M}$ satisfies the condition $\left(3^{\prime}\right)$ for $\mathfrak{A}$ of Fact 4. Therefore,

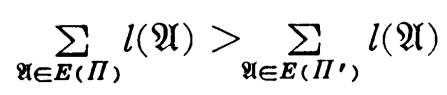

From (i) and (ii), we obtain that $d(\Pi)>d\left(\Pi^{\prime}\right)$. 


\section{Appendix}

The classical natural deduction for which Seldin proved the normalization theorem in [5] was formalized as the intuitionistic logic with Peirce's law. To that system, our reduction-procedure can be applied. The regularity of proofs is defined similarly. For a regular proof $\Pi$, the reduction of $\Pi$ at $\mathfrak{M}$ which is the conclusion of a Peirce's law, say $I$, is defined as follows :
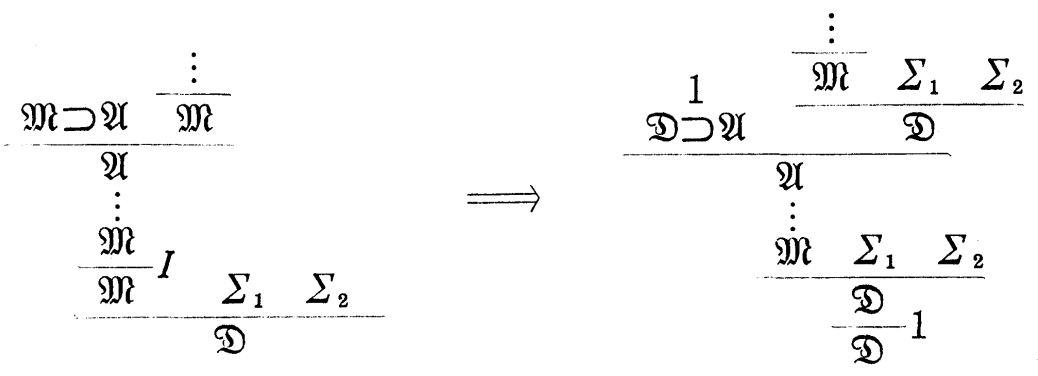

With the appropriate definition of segments, the normalization theorem can be proved similarly to our main issue.

Acknowledgements. I am indebted to the anonymous referee for a careful reading and very helpful comments on earlier versions of the manuscript. I thank Prof. T. Uesu for valuable advices and comments, and Prof. N. Motohashi for suggesting a number of linguistic improvements on earlier versions of the manuscript.

\section{References}

[1] G. Gentzen, Untersuchungen über das logische Schliessen, Mathematische Zeitschrift 39 (1935), 176-210, 405-431.

[2] D. Prawitz, "Natural deduction-A proof theoretical study," Almqvist \& Wiksell, Stokholm, 1965.

[3] D. Prawitz, Ideas and results in proof theory, in "Proceedings of the second Scandinavian logic symposium," North-Holland, Amsterdam, 1971, pp. 235-307.

[4] J.P. Seldin, On the proof theory of the intermediate logic $M H$, Journal of Symbolic Logic 51 (1986), 626-647.

[5] J.P. Seldin, Normalization and excluded middle, I, Studia Logica 48 (1989), 193-217.

[6] G. Stålmarck, Normalization theorems for full first order classical natural deduction, Journal of Symbolic Logic 56 (1991), 129-149.

[7] M.E. Szabo, "The collected papers of Gerhard Gentzen," North-Holland, Amsterdam, 1969. 
Institute of Mathematics

University of Tsukuba

Tsukuba-shi, Ibaraki, 305

Japan

Current Address: Department of Mathematics Hosei University 2-17-1, Fujimi, Chiyoda-ku Tokyo, 102 Japan 